\title{
Digital Humanities Within a Global Context: Creating Borderlands of Localized Expression
}

\section{Amy E. Earhart}

Fudan Journal of the Humanities and Social Sciences

ISSN 1674-0750

Fudan J. Hum. Soc. Sci.

DOI 10.1007/s40647-018-0224-0

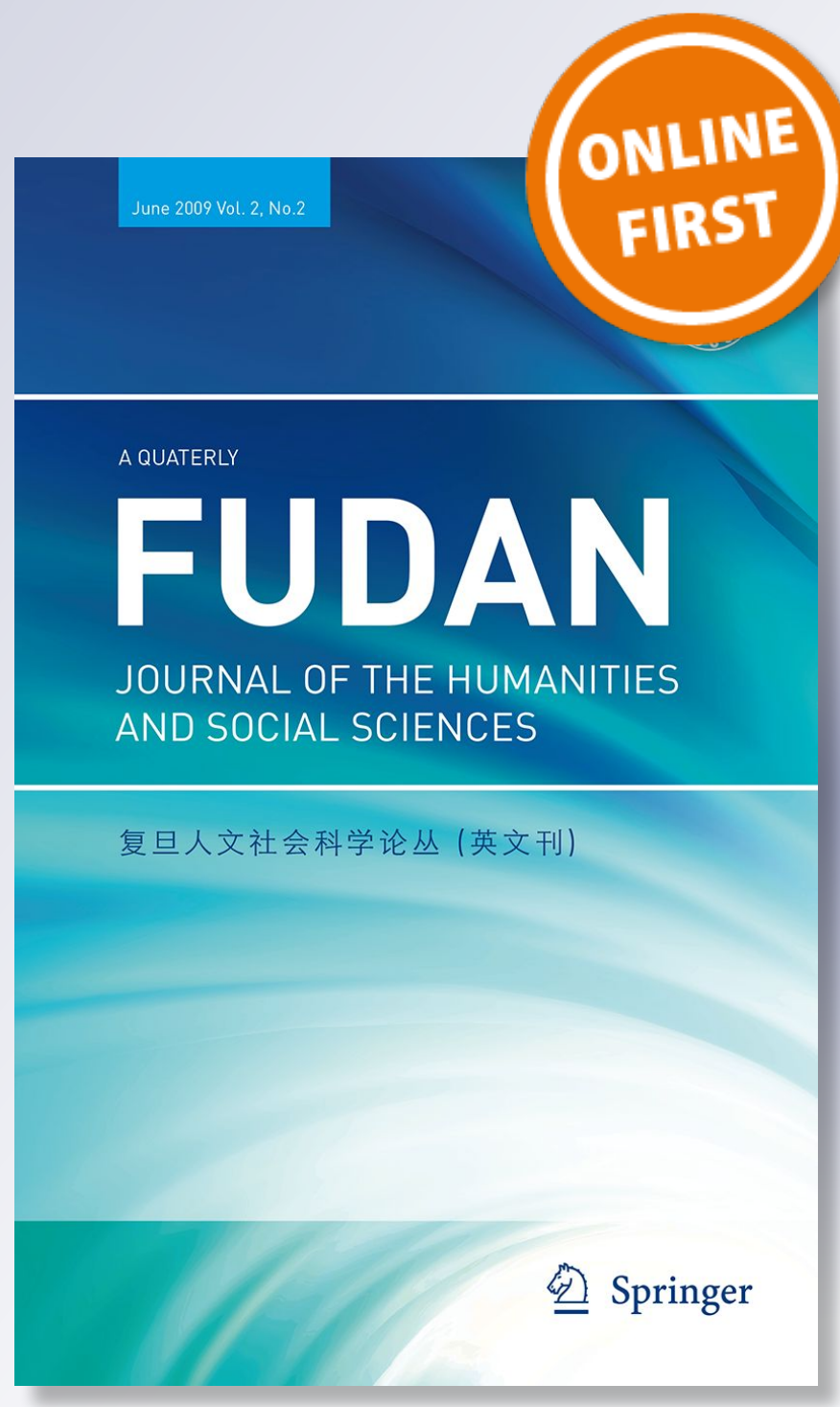


Your article is protected by copyright and all rights are held exclusively by Fudan University. This e-offprint is for personal use only and shall not be self-archived in electronic repositories. If you wish to self-archive your article, please use the accepted manuscript version for posting on your own website. You may further deposit the accepted manuscript version in any repository, provided it is only made publicly available 12 months after official publication or later and provided acknowledgement is given to the original source of publication and a link is inserted to the published article on Springer's website. The link must be accompanied by the following text: "The final publication is available at link.springer.com". 


\title{
Digital Humanities Within a Global Context: Creating Borderlands of Localized Expression
}

\author{
Amy E. Earhart ${ }^{1}$
}

Received: 19 February 2018/ Accepted: 22 March 2018

(C) Fudan University 2018

\begin{abstract}
As scholars have begun the digitization of the world's cultural materials, the understanding of what is to be digitized and how that digitization occurs remains narrowly imagined, with a distinct bias toward North American and European notions of culture, value and ownership. Humanists are well aware that cultural knowledge, aesthetic value and copyright/ownership are not monolithic, yet digital humanities work often expects the replication of narrow ideas of such. Drawing on the growing body of scholarship that situates the digital humanities in a broad global context, this paper points to areas of tension within the field and posits ways that digital humanities practitioners might resist such moves to homogenize the field. Working within the framework of border studies, the paper considers how working across national barriers might further digital humanities work. Finally, ideas of ownership and/or copyright are unique to country of origin and, as such, deserve careful attention. While open access is appealing in many digital humanities projects, it is not always appropriate, as work with indigenous cultural artifacts has revealed.
\end{abstract}

Keywords Digital humanities · Global · Borderlands · Transnational

As scholars have begun the digitization of the world's cultural materials, the understanding of what is to be digitized and how that digitization occurs, of how we utilize technology, of infrastructures of academic digital humanities (dh), remains narrowly imagined, with a distinct bias toward North American and European notions of culture, value and ownership. Humanists are well aware that cultural

Amy E. Earhart

aearhart@tamu.edu

1 Department of English, Texas A\&M University, 4227 TAMU, College Station, TX 77843-4227, USA 
knowledge, academic infrastructures and copyright/ownership are not monolithic, yet digital humanities disciplinary structures often expect the replication of narrow ideas of such. Katherine Hayles predicts an entanglement of codes within a global environment, noting that "As the worldview of code assumes comparable importance to the worldviews of speech and writing, the problematics of interaction between them grow more complex and entangled" (2010, 31). The multiplicity of codes as expressed within global environments brings a largely ignored complexity to digital humanities and code studies and necessitates scholarship to interpret and critique such codes. While digital humanities is global, those of us practicing digital humanities continue to work within, to replicate, localized academic structures. While we might have come to terms intellectually with the notion that our scholarship is looking outward, that we are increasingly called upon to view our work within a complex web of global academic conversations, individual academics remain caught within nationally bound structures of academia, making the notion of a globalized construction of scholarship that values disparate forms of digital humanities incredibly difficult.

As digital humanists imagine the ways that our community of scholars across the world might engage, we have the opportunity to construct a collaborative environment that models the best of such interactions. Efforts are well underway. Models range from a big tent approach, an umbrella model that pulls together all such efforts, to a networked set of nodes. Yet, as global interaction among digital humanists grows it has revealed tension regarding the way in which the digital humanities engage with each other. Rather than initiating a one size fits all global model, we need to imagine a global digital humanities that lives in the borderlands, a place of connection and contradiction and, mostly importantly, a place that does not try to centralize itself.

Recognizing that monolithic models of digital humanities are unproductive, digital humanists have begun to discuss how we might create academic infrastructures, such as organizations, conferences and journals, that fully account for the diversity of practice. Early organizations such as GO::DH, Global Outlook::Digital Humanities, are leaders in the expansion of such infrastructure. Developed to "break down barriers that hinder communication and collaboration among researchers and students of the Digital Arts, Humanities, and Cultural Heritage sectors in high, mid, and low income economies" (GO:DH 2017), GO::DH has become a Special Interest Group (SIG) affiliated with the largest digital humanities organization in the world, the Alliance of Digital Humanities Organizations or ADHO. Work by members of GO::DH and others within ADHO has helped to make building "global digital humanities networks" one of the priorities of ADHO. ADHO has also been working to expand membership, constituent organizations and cultural and linguistic difference within their organization. Other co-partners of ADHO include Centernet: An International Network of Digital Humanities Centers, constructed as "an international network of digital humanities centers formed for cooperative and collaborative action to benefit digital humanities and allied fields in general, and centers as humanities cyberinfrastructure in particular." Emphasizing inclusivity, the organization views itself as a "big tent," extending a welcome to all who self-define as digital humanities. While centernet is an international network 
with expansive goals, it remains limited in representation. Many countries that are actively producing digital humanities work, such as India, are not included in the network. Only two centers in Africa are included, though excellent digital humanities work across Asia is underway. Clearly the largest digital humanities organizations in the world are trying to articulate the way by which they might encourage a global discussion of digital humanities, but remain limited in their success.

Digital humanities as a structural entity has coalesced around the ADHO yearly conference. Since 1989 digital humanists have gathered for the annual conference, imagined as international in scope. Originally the conference rotated between North American and Europe, but in order to encourage international participants the conference has begun to meet in wide ranging locations; it has moved from its original Canadian/US/Western Europe locations to greater parts of Europe and the Americas, such as Poland and Mexico. Created under the umbrella of ADHO, the organization includes The European Association for Digital Humanities (EADH); the Association for Computers and the Humanities $(\mathrm{ACH})$, predominantly an Americas organization; Canadian Society for Digital Humanities/Société canadienne des humanités numériques (CSDH/SCHN); centerNet, Australasian Association for Digital Humanities (aaDH); Japanese Association for Digital Humanites (JADH); and Humanistica, L'association francophone des humanités numériques/digitales (Humanistica). Past conference themes have embraced a global digital humanities. The 2012 international digital humanities conference, held at the University of Hamburg, had the auspicious theme of Digital Diversity: Cultures, Languages and Methods. Australia's hosting of the 2015 conference focused on a theme of Global Digital Humanities. The 2018 Digital Humanities Conference held in Mexico City asks for us to consider Bridges/Puentes. The conference is fairly unique among academic conferences in that it is attempting to pull together such a broad group of scholars. There is no other academic conference in the literature, for example, that has the long-term goal of global outreach and has made such strives toward building a global organization.

Digital humanities journals are also focusing on the global digital humanities and have begun to publish papers that engage with the complex issues of how we might define digital humanities in the increasingly broad space and places in which the scholarship is created. Such efforts extend to journals affiliated with ADHO, including DSH: Digital Scholarship in the Humanities (formerly LLC: The Journal of Digital Scholarship in the Humanities), DHQ (Digital Humanities Quarterly) and Digital Studies/Le champ numérique which have featured global issues, such as collections titled "Digital Humanities Without Borders," "Global Outlook::Digital Humanities: Global Digital Humanities Essay Prize," both in Digital Studies/Le champ numérique, and papers that consider a broader global understanding of digital humanities, such as "Corpus-Based Studies of Translational Chinese in English-Chinese Translation" and "Aspect Marking in English and Chinese: Using the Lancaster Corpus of Mandarin Chinese for Contrastive Language Study," both in DSH: Digital Scholarship in the Humanities.

However, the data suggest that we still have a long way to go if we want to be a global organization. Melissa Terras was the first to focus attention on conference 
representation, finding that the conference was attended overwhelmingly by scholars from the USA, Canada and the UK (see Fig. 1).

Concerned about the lack of geodiversity of conference attendance, Terras has continued to track attendance, and her recent work suggests that digital humanities remains imagined as western located (see Fig. 2).

Work by Roopika Risam, Alex Gil, Isabel Galina, Domenico Fiormont, Elika Ortega, Padmini Ray Murray, among other scholars, have called interpretations such as Fig. 2 into question, suggesting that the digital humanities is centered in the Americas and Europe only in the Western imagination, a construct that ignores the broad scope of global digital humanities. Risam notes, "the distribution of DH centers suggests uneven development. The USA and, to a lesser extent, the UK and Canada appear the true centers of DH, while other countries comprise the peripheries" $(2017,378)$. Should we want to broaden the digital humanities to a globally representative field, then we must begin to not only reimagine boundaries, but to construct organizations which decentralize.

Part of the difficulty is that the structures of the largest digital humanities organizations, such as ADHO, remain narrowly focused. A study of the conference authors from 2004 to 2013 shows that conference participation remains unequally distributed (see Fig. 3).

Conference participation is largely formed by the perennial question of how to define the field, with some definitions driving limited globalized membership, so too might structural issues associated with the conference. centerNet and ADHO offer free and reduced cost memberships for joining their entities and, while waiving membership fees does encourage participation, the actual costs associated with attending the Digital Humanities conference, from airfare to lodging costs, remain high. Registration discounts occur by career stage, with staff and students receiving

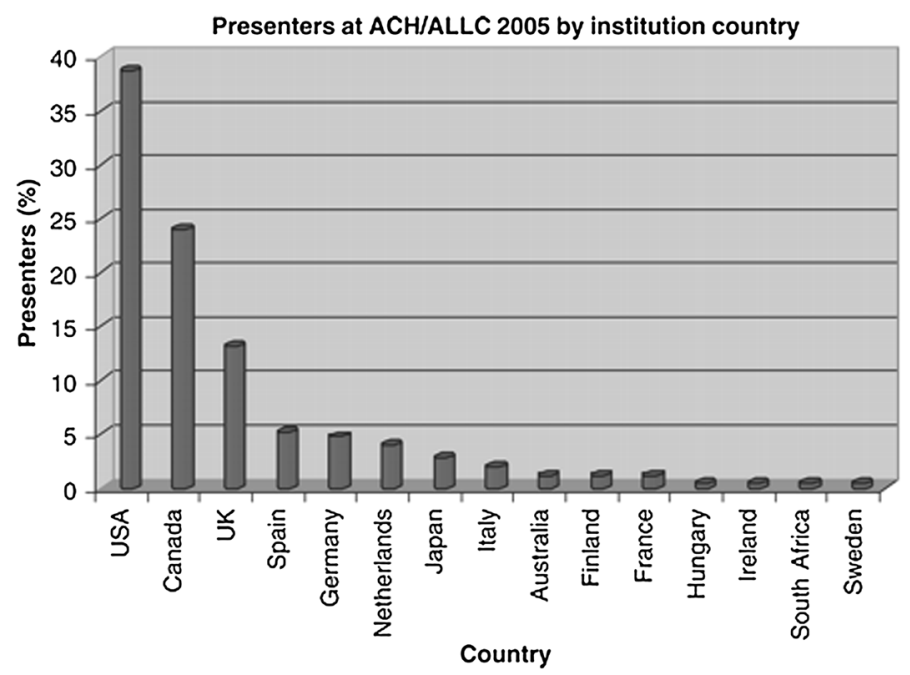

Fig. 1 Presenters at ACH/ALLC 2005 by Institution Country. Terras (2006). Please note that the Digital Humanities Conference was originally titled the ACH/ALLCH conference 


\title{
Quantifying Digital Humanities
}

\author{
- COURTESY OF UCL CENTRE FOR DIGITAL HUMANITIES
}

Digital Humanities research and teaching takes place at the intersection of digital technologies and humanities. DH aims to produce and use applications and models that make possible new kinds of teaching and research, both in the humanities and in computer science (and its allied technologies). DH also studies the impact of these techniques on cultural heritage, memory institutions, libraries, archives and digital culture.

Digital Humanities is difficult to quantify. Here we present all available statistics reguarding individuals and resources, to explore the scope of the field.

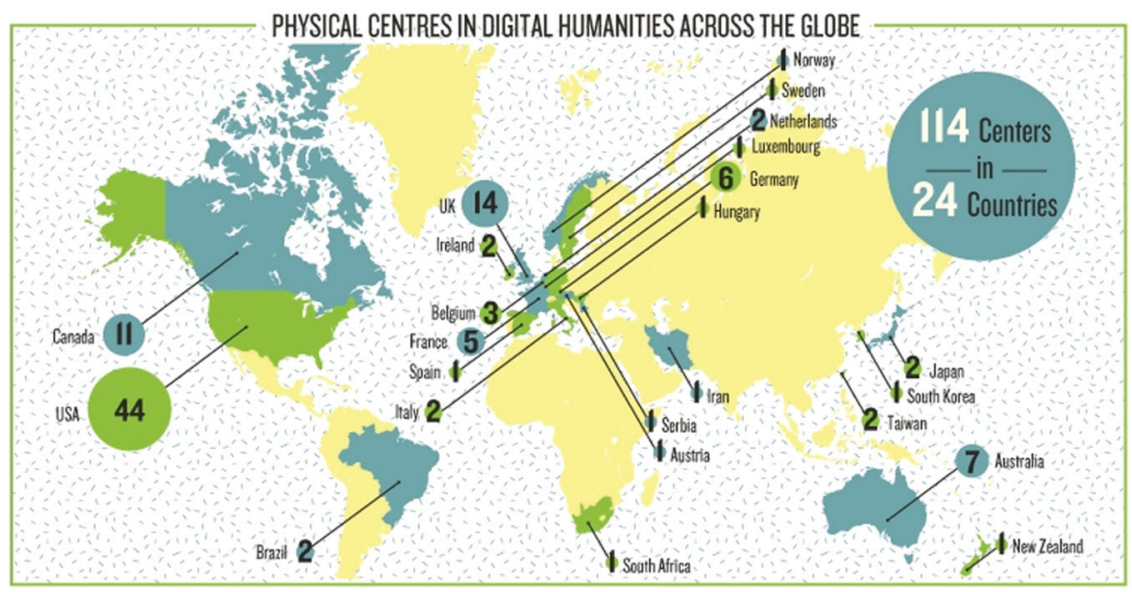

Fig. 2 Quantifying Digital Humanities. Melissa Terras. Infographic: Quantifying Digital Humanities. 2012. Melissa Terras' Blog. http://www.ucl.ac.uk/infostudies/melissa-terras/DigitalHumanitiesInfogra phic.pdf Accessed September 18, 2017

\section{Number of authors per region 2004-2013}

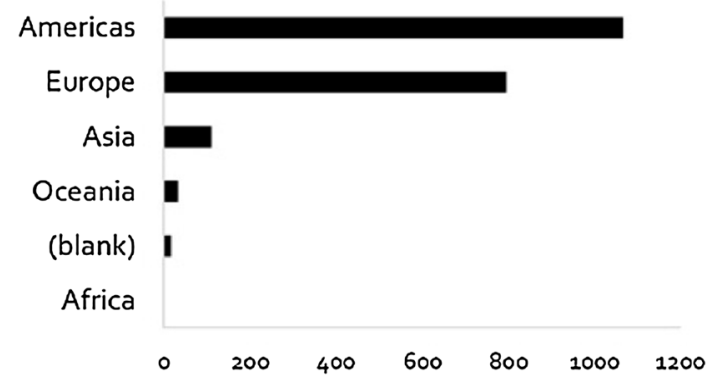

Fig. 3 Number of authors per region 2004-2013. Weingart and Eichmann-Kalwara (2017)

discounted rates, but the organization has not included registration differentiation by region, country or income, leaving those from low-economy counties facing a dramatic challenge. For example, at the 2016 digital humanities conference in Krakow participants from Poland reported that the registration costs of the 
conference were equivalent to a month of salary for lecturers. Though the conference was in their home country, the cost was prohibitive. While some have floated the idea of income-based registration, to date the conference has not responded to a key structural issue that prohibits participation from a broader digital humanities community. The conference has taken positive steps to create a less exclusionary space by holding the 2015 conference in Australia and the 2018 conference in Mexico. Prompted by the 2011 formation of La Red de Humanidades Digitales (RedHD), the 2018 Mexico City conference will be "the first time that the conference will take place in Latin America \& the global south." The shift in locations for Digital Humanities signals an important moment in the history of the organization is largely due to the hard work of organizations like GO::DH and RedHD. However, there remain clear structural barriers to an inclusive global digital humanities.

Algorithmic analysis of digital humanities' structures points to continuing problems in developing a diverse global digital humanities. Scott Weingart's analysis of the yearly ADHO conference has pushed digital humanities to think through how we are constituting ourselves through our conference and our field, revealing the ways that conference participation remains geographically located in the Americas and Europe. ${ }^{1}$ Conference participation limitations also appear in our constituent journals which are likewise publishing articles predominantly clustered around scholars in the Americas and Europe. Telling is an analysis of Digital Humanities Quarterly: $D H Q$ examining co-author networks in the journal from 2007 to 2014 which reveals that the networks remain squarely centered in the Americas, with very little representation beyond Europe (see Fig. 4).

All of this suggests that digital humanities as understood through our organizational entities, digital humanities organizations, conferences and journals, desires to be global but remains merely the imagined global. The domination of the primary modes of disciplinary construction, journals and conferences by the Americas and Europe is a problem in that it is creating a field that runs counter to the described goals of global digital humanities, implying that no matter the imagined global digital humanities, a truly global understanding of an organization or a field is difficult to construct, perhaps even more difficult in the current age of nationalist tensions. There are numerous interventions underway to broaden our representation of global digital humanities, but we remain caught within tensions of an umbrella structure that enforces structures that are often not conducive to the larger representation of digital humanities.

Digital humanities has struggled to articulate a global organization in large part because of originating tensions within the organization construction. Digital humanities, as a field, has struggled to articulate what is included within its rubric, a struggle that remains an open academic question. Tensions within the field have revolved around who's in and who's out, but in a localized context focused on, once again, the Americas and Europe. Reviewing the literature that attempts to define digital humanities reveals that geography has been ignored by scholarship until

\footnotetext{
${ }^{1}$ See dh quantified for a list of scholars invested in collecting information of the community: http:// scottbot.net/dh-quantified.
} 


\begin{tabular}{|c|c|c|c|}
\hline United States (56\%) & 口 Germany (2\%) & Japan (1\%) & Spain $(<1 \%)$ \\
\hline Canada (17\%) & Ireland (2\%) & Norway (1\%) & Mexico (<1\%) \\
\hline U United Kingdom (9\%) & Sweden (2\%) & Portugal $(<1 \%)$ & \\
\hline Netherlands (3\%) & New Zealand (1\%) & Denmark (<1\%) & \\
\hline Australia (3\%) & Italy $(1 \%)$ & Erance $(<1 \%)$ & \\
\hline
\end{tabular}

Fig. 4 "Co-Author Network for Digital Humanities Quarterly: 2007-14." de la Cruz et al. (2015)

recent interventions. Such scholarly constructions of digital humanities which view digital humanities as naturalized within a European and Americas structure has led to current limitations of the field. As O'Donnell et al. make clear, our current representation of digital humanities moves along clear lines of demarcation, whether economic, linguistic or geographic $(2016,493)$. The centering of digital humanities in this manner has created an "unproductive dichotomy of center and periphery," leading to a call for a resistance to such structures through a creation of a regional or local digital humanities (Gil and Ortega 2016, 23). For example, Alex Gil's "Around DH in 80 Days" project resists the limited centering of digital humanities, instead revealing the diversity of global digital humanities projects (see Fig. 5).

The diversification of digital humanities, the struggle to create an organizational entity that inclusively represents a global digital humanities, will continue to occur through ADHO and its affiliated conference and journals, but the organizational structures currently remain resistant to a more globally imagined digital humanities. Because of this, we might ask whether ADHO is actually the mechanism to bring about global digital humanities. As the organization has grown, there has been an almost de facto understanding that it should be the center for global dh. But the centering of digital humanities in an organization that has arisen out of western academic structures will, I argue, always struggle to imagine how to construct a truly representative field. A better question might be whether we can construct an alternative mechanism that accurately represents all the different ways that digital humanities is practiced in a global environment.

The rejection of an umbrella or big tent organization in which to coalesce a global digital humanities is born out of an analysis of the way that geographic, economic, cultural and structural approaches to academic discipline impact our interactions in the larger digital humanities. During the research and writing of Traces of the Old, Uses of the New: The Emergence of Digital Humanities (2015) I came to understand that providing one definition of the digital humanities was dependent upon a stable infrastructure from which the practice developed. The definition of digital humanities within the Americas is dependent upon an academia that is increasingly defunded and deprofessionalized, driving a digital humanities that is interested in an entrepreneurially based startup model of digital humanities. This is not so for other localized digital humanities practices, yet dh organizations like ADHO continue to imagine digital humanities with a distinct bias toward North American and European notions of culture, value and ownership. O'Donnell et al. rightly argue that this view of digital humanities is predicated on viewing the development of a global digital humanities "as an opportunity for transferring 
Around DH in 80 Days $\quad$ About Credits

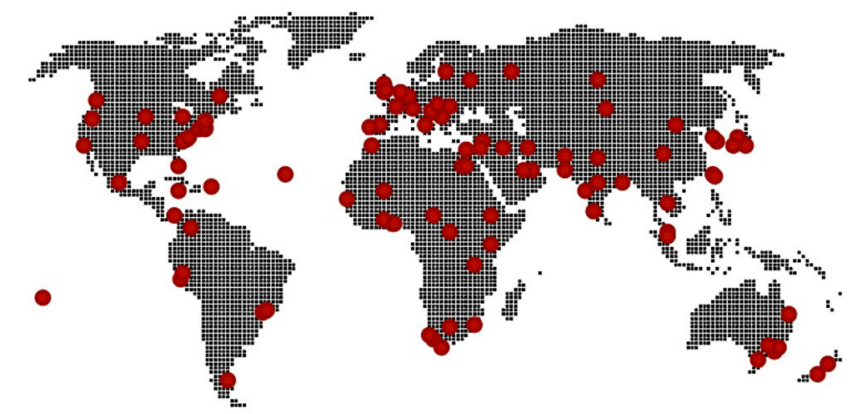

The Full Journey.

Around $\mathrm{DH}$ in 80 Days

Contact us
Oelotroalex/arounddh

\#arounddh
This work is licensed under a Creative Commons

Attribution 3.0 United States License.

Fig. 5 “Around DH in 80 Days." Gil (2014)

knowledge, experience, and access to infrastructure from a developed North to an underdeveloped South" $(2016,496)$. Rejecting this, the authors call for an approach that "is far more about developing understanding than merging practice," and they turn to "supra-networks that transcend national, linguistic, regional and economic boundaries" $(2016,496)$. I'd like to quibble with the use of networks as the way by which we should represent the interaction of the various global representations of digital humanities. The notion of an overarching system that is built from nodes, is not that different than how ADHO and its constituent conference imagines itself, a model that ignores the very real institutional and cultural divides that are always with us. In many ways, a supra-network is a slightly shifted replication of the long understood big tent digital humanities and, ultimately, a failed model.

Digital humanities is an amorphous and fluid concept or practice, particularized in various disciplines, national contexts and even local environments, but the field is represented as a coherent body of practice by intact structures that include the annual digital humanities conference, the various global organizations that form $\mathrm{ADHO}$, and even journals published by the various societies. The digital humanities, as represented by the yearly international conference, is a digital humanities which ignores the borders of practice that masks areas of dissension and normalizes the field to a particular form without contour. However, the center does not hold and recent conferences have featured ruptures, revealing the false constructedness of a coherent digital humanities. Structuring the global digital humanities as a "big tent" hides the way that such a representation seeks "sameness" in practice. A counternarrative that provides a more inclusive understanding of global digital humanities is one that turns to specificity. While some may see the segmentation of digital humanities as counterproductive, I argue 
that digital humanities must be particularized because $\mathrm{dh}$, as enacted, is so broad, diffuse and flexible that a generalized definition does not adequately address the various digital approaches currently in use nor how certain humanities fields are being altered by digital practice. A far more productive understanding of our collective histories is to identify the borders of practice and to look for disciplinary overlaps that benefit all partners.

A specificity of global digital humanities' practices is best understood in the framework of what Gloria Anzaldua has called the borderlands in her crucial work Borderlands|La Frontera (1987). Anzaldua's framework allows us to examine the impact of cultural representations of digital humanities within larger frameworks of power, including the economic, cultural and power dynamics that impact the production of scholarship. While Anzaldua is writing prior to the digital turn and code studies scholarship, her work is prescient. Examining the code shifting of language, Anzaldua argues that language codes provide a way to examine the complexity of networked interfaces of communication and a way of understand how cultural identity is impacted by power dynamics of such code. Anzaldua's focus on code switching, defined in her book as language switching or "The switching of 'codes' ...from English to Castillian Spanish to the North Mexican dialect of TexMex to a sprinkling of Nahuatl to a mixture of all of these," produces great cultural upheaval. This "language of the Borderlands" is ever shift and changing and "There, at the juncture of cultures, languages cross-pollinate and are revitalized; they die and are born" (1987, Preface). While Anzaldua situates her discussion of borderlands in the geographic specificity of the Texas/Mexico border, her theorization of power between multiple cultural codes might be extended to our understanding of digital humanities. Roopika Risam echoes such an extension of code switching when she calls for $\mathrm{DH}$ accents, a recognition of the multiple languages, both "linguistic and computational" as the formation of $\mathrm{dh}(\mathrm{s})$ (2017, 381). To Risam, the multiple accents of digital humanities must be "understood in a broader ecology of 'accents' that inflect practices, whether geography, language, or discipline," providing a model that makes sense of and values the broadness of digital humanities, rather than contains such diversity within a limited framework (2017, 382).

Key to understanding the way that localized digital humanities interact within a global framework is to evaluate the contingent power structures. Anne Donadey notes, "Discrete fields of knowledge can be seen as being separated by disciplinary borders; the interdisciplinary and comparative areas where they meet and are brought together can be viewed as borderland zones in which new knowledge is created, sometimes remaining in the borderland, sometimes becoming institutionalized into a different field of knowledge with its own borders" (2007, 23-24). The importance of borders is not in the separation, though indeed that is in play, but the meeting points, which provide productive tensions that bring forth new knowledge.

Focusing on resistance, as Donadey puts it, avoids the flattening of "the concept of borderlands that would erase its historical and cultural grounding by turning it into a disembodied metaphor" $(2007,23)$. The borderlands stand in opposition to big tent representations of cultural connection. To embrace a borderlands understanding of global digital humanities is to respect localized practices and to 
embrace points of context rather than a homogenized centrality. As Anzaldua reminds us, "A borderland is a vague and undetermined place created by the emotional residue of an unnatural boundary. It is in a constant state of transition" $(1987,3)$. The continual renegotiation of points of connection is productive and ever shifting. Rather than attempting to stabilize such moments, border theory seeks fluidity and destabilization as a means of new knowledge production. Viewing the global digital humanities within a border theory model rather than a big tent or umbrella formulation, one journal or one conference, allows scholars to seek those points of contact while understanding how the power dynamics of digital humanities have come to create points of contention.

Crucial to respecting the integrity of localized digital humanities is a careful examination of our assumptions about technology use in digital humanities projects. GO::DH has supported "minimal computing" approaches as a way to rethink the way that many western digital humanities projects center technology innovation. Based on discussions in 2014 with digital humanists in Cuba, those associated with GO::DH, led by Alex Gil, recognized that computing needs in various localized environments might benefit from what Ernesto Oroza calls the "architecture of Necessity" (Gil and Ortega 2016, 29). GO::DH has defined "minimal computing" as that which "simultaneously capture(s) the maintenance, refurbishing, and use of machines to do DH work out of necessity along with the use of new streamlined computing hardware like the Raspberry Pi or the Arduino micro controller to do DH work by choice. This dichotomy of choice versus necessity focuses the group on computing that is decidedly not high-performance and importantly not first-world desktop computing" (GO::DH 2017). While we continue to need to explore how technologies benefit our research questions, we cannot ignore more minimal computing approaches that are often the most innovative and expansive within our field. The bias toward highly robust, often expensive, technologically centered projects as the gold standard for dh also creates a centered field that actively ignores the work occurring in some parts of global digital humanities. To best move forward, we need to return to a multiplicity of approaches that allows for scholarship to recenter technology, and we must resist the creation of rigid borders of academic disciplinarity that effectively shuts down the possibilities of global digital humanities interchange. To proceed in a non-policed borderlands, we must resist a tyranny of technology. Frames for our community interaction must be fluid and non-centralized. They must be evolving. To enable the productive friction between communities, we might begin to see our fields as less about connective nodes and networks and more focused on transnational understandings of disconnecting nodes.

Border theory expands our methodologies and our approaches, rejecting a narrow understanding of digital humanities. It allows us to rethink the way that our own scholarship has been colonized and limited, particularly through models of ownership. A tenet of digital humanities in the Americas, for example, has focused around issues regarding ownership of scholarship, with faculty increasingly asserting control over their own labor and their ability to disseminate it freely, as open access (oa) materials, to an audience apart from or in parallel with more traditional structures of academic publishing. Key to defining the digital humanities 
then is that our scholarship is increasingly public. Matthew Kirschenbaum notes that "Whatever else it might be then, the digital humanities today is about a scholarship (and a pedagogy) that is publicly visible in ways to which we are generally unaccustomed, a scholarship and pedagogy that's bound up with infrastructure in ways that are deeper and more explicit than we are generally accustomed, a scholarship and pedagogy that is collaborative and depends on networks of people and that lives an active, 24/7 life online" $(2012,60)$. The public digital humanities and the accompanying push for open access are central to the way that many digital humanists situate their scholarship. However, to fully encompass all expressions of digital humanities, we must also think carefully about issues of ownership, which many in digital humanities have expressed in limited western contexts such as copyright.

As we move toward a model of interchange and exchange of globalized digital scholarship, the understanding of ownership and open access must be carefully examined and complicated. The dominance of models of open access in the Americas has been critiqued by a growing number of scholars, with particular attention to this issue from scholars who work with indigenous communities and knowledges. Kim Christen, for example, has produced scholarship and innovative digital tools to address issues of ownership and openness that are centered on indigenous knowledge structures. Her work recognizes that the digital archiving process has deep roots in museum and library collections' problematic pasts and that many indigenous communities' have had their intellectual production exploited by colonizers. As Christen notes, "The colonial collecting project was a destructive mechanism by which Indigenous cultural materials were removed from communities and detached from local knowledge systems" $(2015,2)$. In response, Christen has developed a content management system (CMS), Mukurtu, that allows for sophisticated control of the materials within the CMS, demarcating the viewing of digital objects through localized understandings of what should be seen and what should not be seen and forcing the user to understand that there are certain objects or ideas that are not open to all. ${ }^{2}$

While Christen's work explicitly targets indigenous groups, her thinking about what should be seen and what should not be seen models best practices that we must extend into our conception of the global digital humanities. At the 2017 Montreal Digital Humanities meeting the "Copyright, Digital Humanities, and Global Geographies of Knowledge" panel considered this important issue. The discussion of copyright practices in various countries during the panel revealed the very limited understanding of the topic within the larger collective who attended the conference. Isabel Galina Russell's remarks focused on copyright in Latin America, with her particular expertise focused on Mexico. Galina Russell emphasized that "Latin America distinguishes itself from other regions of the world in that scientific information belongs to all" (2017). Recognizing that few for profit academic commercial publishers exist in Latin America, Galina Russell argues that "there is a

\footnotetext{
${ }^{2}$ See Kimberly Christen. "On Not Looking: Economies of Visuality in Digital Museums" in The International Handbooks of Museum Studies: Museum Transformations, First Edition. Ed. Annie E. Coombes and Ruth B. Phillips. Oxford: John Wiley \& Sons, Ltd. Oxford Press, 2015: 365-386. $365-3666$.
} 
generalized idea that knowledge produced in the university belongs to all, it is a common good provided to the country," negating copyright and shifting ownership of academic production to the public (2017). This conception of ownership stands in stark contrast to the way that ownership has functioned within the types of structures set up by the western for profit academic publishers and that many dh scholars see as central to oa initiatives. In the same panel, Padmini Ray Murray discussed the copyright lawsuit brought against Shyam Singh, the owner of a small Indian shop producing course packs for students at a local university, who was sued by several leading academic presses. Murray points out that the case revealed the way that assumptions of copyright elided national boundaries and attempted to apply western understandings of ownership on scholarly work. At the same time that the lawsuit negated copyright rules of the Indian state, it also selectively ignored US and UK copyright rules with the desire to further enforce western ideas of ownership. In response to the supposed copyright violations, the lawsuit "sought to ban all course packs, including those that observe the US definition of fair use, i.e., excerpts comprising less than $10 \%$ of the whole text" (2017). At the same time the legal challenge ignored "Section 52 of the Indian Copyright Act $<$ that $>$ permits "fair dealing' with the purpose of research, as well as permitting any copyrighted work to be used for the purpose of educational instruction" (2017). Situating copyright law neither in Indian or the west, the lawsuit was written as nationless, boundary less, centered only on the effort to end the exchange of information. Both papers point to the complications of thinking about ownership and knowledge as equivalent forms across cultures and nations. While we might value open access in the digital humanities, not all producers of knowledge will accede to openness. Instead we must, once again, develop structures that see knowledge as culturally defined and controlled. By valuing the localized understanding of knowledge and knowledge production, we situate the global digital humanities within a productive nexus of borders.

Instead of insisting that we encapsulate all practices of digital humanities within a big tent or a centralized structure, we should instead view ADHO and its conferences and journals as important, but not central, meeting spaces for digital humanists. Rather than seeing ADHO as the center, we should encourage a global digital humanities that works on the borderlands, with localized expressions of scholarship that reinvigorate through exchange. Rejecting the "dualistic thinking in the individual and collective consciousness" is a struggle, as Anzaldua argues, but it is the only way that we might move beyond binaries that are currently in place, whether technologically advanced/primitive, east/west, or low income/high income (1987, 422). Resisting the homogenization of scholarly methods, questions, outcomes, production and ownership is the only way to develop a truly robust global digital humanities. 


\section{References}

Anzaldua, Gloria. 1987. Borderlands/La Frontera. San Francisco: Aunt Lute Book Company.

Centernet: An International Network of Digital Humanities Centers. 2017. https://dhcenternet.org/about. Accessed 15 Aug 2017.

Christen, Kimberly. 2015. Tribal Archives, Traditional Knowledge, and Local Contexts: Why the 's' Ma Ers. Journal of Western Archives 6(1): 1-19.

de la Cruz, Dulce Maria, Jake Kaupp, Max Kemman, Kristin Lewis, and Teh-Hn Yu. 2015. Mapping Cultures in the Big Tent: Multidisciplinary Networks in the Digital Humanities Quarterly. https:// jkaupp.github.io/DHQ/coursework/VisualizingDHQ_Final_Paper.pdf. Accessed 10 Aug 2017.

DH2018: Mexico City. Dh 2018 (blog) 2018. https://dh2018.adho.org/en/. Accessed 10 Aug 2017.

Donadey, Anne. 2007. Overlapping and Interlocking Frames for Humanities Literary Studies: Assia Djebar, Tsitsi Dangarembga. Gloria Anzaldua. College Literature 34(4): 22-42.

Earhart, Amy E. 2015. Traces of the Old, Uses of the New: The Emergence of the Digital Literary Studies. Ann Arbor: University of Michigan Press.

Galina Russell, Isabel. 2017. Presentation, Panel on Copyright, Digital Humanities, and Global Geographies of Knowledge. Presented at the Digital Humanities 2017, Montreal, Canada.

Gil, Alex. 2014. Around DH in 80 Days. Around DH in 80 Days (blog). http://www.arounddh.org. Accessed 10 Aug 2017.

Gil, Alex, and Elika Ortega. 2016. Global Outlooks in Digital Humanities: Multilingual Practices and Minimal Computing. In Doing Digital Humanities: Practice, Training, Research, ed. Constance Crompton, Richard J. Lane, and Ray Siemens, 22-34. London: Routledge.

Global Outlook::Digital Humanities. 2017. http://www.globaloutlookdh.org. Accessed 10 Aug 2017.

Hayles, Katherine. 2010. My Mother Was a Computer: Digital Subjects and Literary Texts. Chicago: University of Chicago Press.

Kirschenbaum, Matthew. 2012. What is Digital Humanities and What's It Doing in English Departments? In Debates in the Digital Humanities, ed. Matthew Gold, 3-11. St. Paul: U Minnesota P.

Membership. ADHO (blog). 2018. https://adho.org/faq. Accessed 10 Aug 2017.

O'Donnell, Daniel Paul, Katherine L. Walter, Alex Gil, and Neil Fraistat. 2016. Only Connect: The Globalization of the Digital Humanities. In A New Companion to the Digital Humanities, ed. Susan Schreibman, Ray Siemens, and John Unsworth, 493-510. Malden, MA: Wiley Blackwell.

Pannapacker, William. 2009. The Brainstorm Blog: The Chronicle of Higher Education Online.

Ray Murray, Padmini. 2017. Presentation, Panel on Copyright, Digital Humanities, and Global Geographies of Knowledge. Presented at the Digital Humanities 2017, Montreal, Canada.

Risam, Roopika. 2017. Other worlds, other DHs: Notes towards a DH Accent. Digital Scholarship in the Humanities 32(2): 377-384.

SIGs: ADHO Special Interest Groups (SIGs). 2017. ADHO (blog). http://adho.org/sigs. Accessed 3 Nov 2017.

Terras, Melissa. 2006. Disciplined: Using Educational Studies to Analyse 'Humanities Computing'. Literary and Linguistic Computing 21(2): 229-246.

Terras, Melissa. 2011. Quantifying Digital Humanities. UCL Centre for Digital Humanities. http://blogs. ucl.ac.uk/dh/2012/01/20/infographic-quantifying-digital-humanities/. Accessed 5 Nov 2017.

Weingart, Scott B., and Nickoal Eichmann-Kalwara. 2017. What's Under the Big Tent? A Study of ADHO Conference Abstracts. Digital Studies/Le Champ Numerique 7: 6. https://doi.org/10.16995/ dscn.284/.

Amy E. Earhart is an Associate Professor in the Department of English at Texas A\&M University. She is the author of Traces of the Old, Uses of Old: The Emergence of Digital Literary Studies (2015) and coeditor of The American Literature Scholar in the Digital Age (2010). She is the author of various books and chapters in venues including Debates in Digital Humanities, Textual Cultures and the Humanities and the Digital, among others. 